\title{
Stability optimal control for liquid tank under transverse excitation
}

\section{Zhixin Yu, Jie Li*, Fuguang Dai, Shaosong Li and Xinxin Cheng}

School of Mechatronic Engineering, Changchun University of Technology, Changchun 130000, China

Email: yuzhixin@ccut.edu.cn

Email: Jasonliccut@163.com

Email: dai_fuguang@163.com

Email: lison19860922@163.com

Email: 289125828@qq.com

*Corresponding author

\begin{abstract}
This paper studies the problem of liquid tank stability control on account of the interaction between liquid sloshing and vehicle in emergency obstacle or turning. The dynamic fluid sloshing model within the tank is modelled using governing equations with potential flow theory, combined with the rigid model of semi-trailer; the liquid sloshing model is integrated into the vehicle model. We compare the stability influence of tank with the same mass of liquid cargo and solid cargo in fishhook. With this unsteady state factor, we designed the optimal control strategy and co-simulation in Matlab/Simulink and TruckSim. Simulation results show that the proposed control approach is effective in rollover prevention of liquid tank under transverse excitation.
\end{abstract}

Keywords: tank trucks; liquid sloshing; filling ratio; optimal control; differential braking.

Reference to this paper should be made as follows: Yu, Z., Li, J., Dai, F., Li, S. and Cheng, X. (2020) 'Stability optimal control for liquid tank under transverse excitation', Int. J. Vehicle Safety, Vol. 11, No. 3, pp.214-228.

\section{Introduction}

It is known that fluid oscillations in partially-filled tank vehicles arising from road or manoeuvre-induced excitations cause undesirable hydrodynamic forces and moments of significant magnitude. As the capacity is large, centre of mass is higher than the wheelbase; it is prone to rollover and other unstable accident, which can be easily accompanied by leakage, combustion and explosion of oil products. The design and analysis of liquid tank thus necessitates consideration of dynamic slosh loads due to the transient motion of the fluid cargo. In order to reduce the adverse effect of fluid sloshing on stability of tank vehicles, it is essential to analyse fluid sloshing characteristics and formulate effective control strategy to make sure the vehicle is stable. 
Fluid sloshing in moving containers has been widely investigated using different methods. According to Kang et al. (2000) interactions between the sloshing liquid and the vehicle system, however, have been mostly limited to quasi-static fluid slosh models, which neglects the contributions due to dynamic sloshing. Applications of the dynamic slosh to the vehicle models, however, have met limited success due to high computational demands of the Computation Fluid Dynamic (CFD) methods and the need for elaborate data transfers for coupled fluid-vehicle simulations. Cheli et al. (2013) and Yan et al. (2009) investigated steering and braking performance of partially filled tank trucks by coupling the multi-body vehicle models with the Fluent CFD software. Alternatively, a number of mechanical analogous models have been proposed to describe liquid oscillations in moving containers, which can be directly integrated to the vehicle models to perform coupled fluid vehicle dynamic analyses in an efficient manner. Identification of the mechanical model parameters, however, requires prior CFD simulation, experimental measurements or analytical solutions (Salem et al., 2009).

The structure optimisation of liquid tank can improve its stability to a certain extent; however the effect is far less than the active control strategy of vehicle. The major active control is differential braking. A number of studies have proposed different vehicle dynamic control systems using differential braking. These have shown their superior effectiveness in realising improved handing, stability limits and tracking the desired yaw response through simulations and road tests (Chen and Tomizuka, 2000; Zhao et al., 2017; Bin et al., 2015). Goodarzi et al. (2009) designed an integrated control system for stability enhancement of articulated vehicles considering both the yaw and roll moments to improve vehicle stability. The first-layer controller was used to determine the corrective yaw and roll moments, while the second-layer controller determined braking forces distribution among the wheels to achieve the desired yaw moment.

On the other hand, some efforts have also been made towards developments in active trailer steering systems to improve directional response and yaw stability of the combinations. Oreh et al. (2013, 2014), Song et al. (2007), Ashtiani et al. (2014) investigated directional control of articulated heavy vehicle using active steering control. Three state variables, namely the tractor's yaw rate, the tractor's lateral velocity and the articulation angle, were considered as the controlled target responses. The study presented the design of sliding mode and $L Q R$ controller, and compared the relative performance in terms of improvements in the directional stability limit under various manoeuvres. Azad et al. (2009) conducted a survey on various stability enhancement strategies for articulated steering vehicles and concluded that the previous studies on the stability and control of articulated steering vehicles mainly focused on the snaking stability.

Reported studies have invariably shown that active safety systems can yield significant improvement in handing and stability performance of articulated vehicle. However, the designs of control strategies need careful consideration with regard to selection of control objective and how the action is realised. In this paper, an active control strategy considering the braking of both tractor and semi-trailer and the effectiveness of anti-rollover control is evaluated in a co-simulation environment involving Matlab / Simulink and TruckSim.

The rest of the paper is structured as follows. The fluid slosh characteristics are analysed in Section 2. The influence factor of sloshing forces is got in Section 3. The effective of fluid slosh on vehicle is analysed in Section 4. In Section 5, the proposed active braking control scheme is formulated together with synthesis of the $L Q R$ yaw 
moment controller to reduce the yaw rate errors. The braking torque distribution scheme is subsequently proposed to achieve desired yaw moments. Simulation results are obtained under extreme conditions to illustrate effectiveness of the propose active braking control strategy in terms of the rollover prevention, while the major findings of the study are summarised in Section 6.

\section{Liquid sloshing analysis}

The shapes of tank vehicles are usually oval, round or convex, when the internal pressure of the fluid is large, a round section tank can be used to ensure the strength of the tank. In this paper, we consider a partially-filled cylindrical container of radius $R$ and length $L$, where $L$ is much larger than $R$. The influence of both ends of the tank can be ignored, analysing the unit length of cylinder without considering vertical motion. "O", as shown in Figure 1. The Cartesian coordinate system $x y z$, located at top of the tank, is used with $x$ axis along the motion of tank truck, $y$-axis to the left side of the tank, and $z$-axis directed upward. In this figure, $S$ represent the fluid free-surface, $\Omega$ and $\Sigma$ are the fluid area and wetted surface area of the tank wall, the liquid under fill level $h_{0}$ is measured with respect to the tank roof, $\theta_{0}$ is the angle between the line which free liquid surface and tank interconnect with point $O$ and negative half axis of $Z$.

Figure 1 Tank filling system coordinate

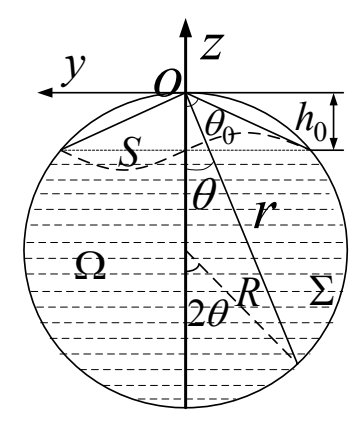

Assuming inviscid, incompressible and irrotational flow, as fluid satisfy continuity equation in fluid aero, impermeable condition on rigid tank wall and kinematics and dynamics equation on free liquid surface. The control equation can be expressed as:

$$
\begin{cases}\nabla^{2} \varphi=0 & \in \Omega \\ \frac{\partial \varphi}{\partial n}=0 & \in \Sigma \\ \frac{\partial \varphi}{\partial n}=\frac{\partial \eta}{\partial t}, \frac{\partial \varphi}{\partial t}+g \eta=0 & \in S\end{cases}
$$

During the liquid sloshing, the velocity potential is decomposed into two parts: the inertia component of the liquid and the dynamic pressure component. The potential $\varphi_{u}(r, \theta, t)$ 
representing the rigid body motion of the fluid, which is same as the potential of the container motion; and the potential $\varphi_{s}(r, \theta, t)$ representing the liquid motion relative to the container, which is the potential of sloshing fluid. The velocity potential can be expressed as:

$$
\varphi=\varphi_{u}(r, \theta, t)+\varphi_{s}(r, \theta, t)
$$

Both the potentials $\varphi_{s}(r, \theta, t)$ and $\varphi_{u}(r, \theta, t)$ satisfy equation (1), for inertia potential, it can be expressed as:

$$
\varphi_{u}(r, \theta, t)=\dot{Y} r \sin \theta
$$

where $\dot{Y}$ is the speed of $Y, R$ is the radius of tank. Here the natural slosh modes, assume $\varphi=i \sigma \Phi(r, \theta) e^{i \sigma t}, \eta=H(r, \theta) e^{i \sigma t}$ and substituting $\varphi$ and $\eta$ into equation (1), the equation (1) can be rewritten as follows:

$$
\left\{\begin{array}{l}
\nabla^{2} \varphi=0 \\
\frac{d \phi}{d n}=0 \\
\frac{d \phi}{d n}=H, H=\frac{\omega^{2}}{g} \phi
\end{array}\right.
$$

where $\phi$ refers to shake mode function, $H$ refers to wave height mode function, $\omega$ refers to fluid slosh characteristic frequency, the characteristic frequency and mode function of equation (4) can be solved by Galerkin.

The characteristic mode is the inherent property of sloshing liquid, it is also applicable for the force sloshing mode, so $\phi, \eta$ can be expressed by the combination of $\phi$ and $H$.

$$
\varphi=\sum_{i=1}^{N} \dot{q}_{i}(t) \Phi_{i}, \eta=\sum_{i=1}^{N} q_{i}(t) H_{i}
$$

where: $q_{i}(t)$ is generalised coordinates. Substituting equation (5) into equation (1), then Bernoulli integral equation (1) and eliminating $\eta$, the final equation can be written as:

$$
\sum_{i=1}^{N} \Phi_{i}\left[\ddot{q}_{i}(t)+q_{i}(t) \sigma_{i}^{2}\right]+v_{0} \cdot r=0
$$

Multiplying $\rho H_{i}$ both side of equation (6) and integrating it on the free fluid surface, the following infinite system of second order ordinary linear differential equation is obtained:

$$
M \ddot{Q}+K Q=-\xi \ddot{Y}
$$


where

$$
\begin{aligned}
& M_{i j}=\int_{s} \rho H_{j} \phi_{i} d S, K_{i j}=\int_{s} \rho \sigma_{i}^{2} H_{j} \phi_{i} d S, \\
& \xi=\int_{s} \rho H_{j} \sin \theta d S, H=\frac{\sigma^{2}}{g} \phi, d S=\frac{h_{0}}{\cos ^{2} \theta} d \theta
\end{aligned}
$$

The system of equation (7) express the dynamic equilibrium of the system, where $Q$ is the matrix of unknown generalised coordinates, $M$ and $K$ may be considered as the mass and stiffness matrices of system, respectively, and $\xi$ is the matrix expressing the contribution of external excitation on the dynamic equilibrium.

Since the numerical method is based on the potential flow, and the assumption is there isn't energy dissipation, a ratio damping needs to be introduced in equation (7) to account for the damping effect, the ratio damping can be expressed as:

$$
C=\partial_{0} M+\partial_{1} K
$$

where $\partial_{0}$ and $\partial_{1}$ are constants, equation (7) can be rewritten as:

$$
M \ddot{Q}+C \dot{Q}+K Q=-\xi \ddot{Y}
$$

To meet accuracy of the calculation, intercepting a certain order number during calculation, the dynamic pressure potential can be calculated:

$$
\varphi_{s}(r, \theta, t)=\sum_{i=1}^{N} q_{i}(t) \frac{\sin (i t)}{r^{i}}
$$

\section{Fluid transverse sloshing effects analysis}

\subsection{Hydrodynamic pressures and forces}

Once the velocity potential $\varphi$ associated with sloshing is calculated, the hydrodynamic pressure at any location can be computed from the linearised Bernoulli equation and the total horizontal force acting on the container is obtained by an appropriate integration of the pressure on the hemispherical wall. The total hydrodynamic force $F_{h}$ on the container wall is the sum of the inertia force $F_{u}$ and dynamic force $F_{s}$. The container's forces are given by

$$
\begin{aligned}
& F_{u}=\int_{\Sigma}-\rho\left(g z^{\prime}+\frac{\partial \varphi_{u}}{\partial t}\right) \cos (n, \vec{i}) d \Sigma=-M \ddot{Y} \\
& F_{S}=\int_{\Sigma}-\rho\left(g z^{\prime}+\frac{\partial \varphi_{S}}{\partial t}\right) \cos (n, \vec{i}) d \Sigma=-\sum_{i=1}^{N} G_{i} \ddot{q_{i}}
\end{aligned}
$$

and

$$
F_{h}=F_{u}+F_{S}=-M \ddot{Y}-\sum_{i=1}^{N} G_{i} \ddot{q_{i}}
$$


where

$$
G_{i}=\rho 2^{1-i} R^{1-i} Z i L, Z_{i}=\int_{-\theta_{0}}^{\theta_{0}} \frac{\sin (i \theta) \sin (2 \theta)}{(\cos \theta)^{i}} d \theta,(i=1,2, \ldots), \rho
$$

is the fluid mass density.

Since the pressure is always normal to the wall of the container, the total hydrodynamic force direction always passes through the centre of the cross-section of horizontal cylinder.

\subsection{The effect of fill ratio on liquid sloshing}

The fill ratio directly affects the fluid inertia, height of centre of gravity and the load shift potential and the associated force and moment. To quantitatively describe the load of the tank, we define fill ratio $\lambda$ as the ratio of the liquid height to the radius. Liquid density $\rho=580 \mathrm{~kg} / \mathrm{m}^{3}$, the radius of tank $R=1.5 \mathrm{~m}, L=12 \mathrm{~m}$, gives a truncation size $N=4$ of equation (9), analysis the inertial force $F_{u}$, sloshing force $F_{S}$ and total force $F_{h}$. The Figure 2 is the lateral force variation with the fill ratio.

Figure 2 The variation of the lateral force

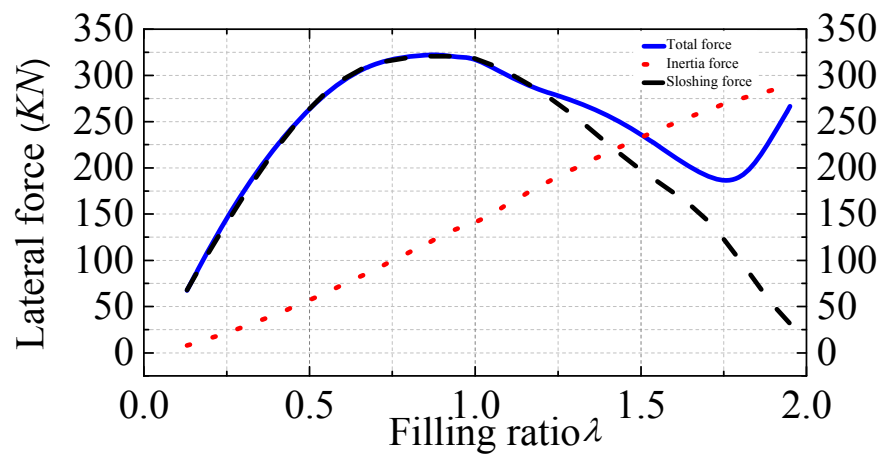

Figure 2 shows that as the fill ratio increases, the inertia force increases, the sloshing force increases first and reaches peak at fill ratio of $\lambda=1$ and then decreases. When the fill ratio is small, the inertial force can be ignored relative to the dynamic force; as the fill ratio increases, parts of fluid which participate sloshing is decreasing, the dynamic force action decreases, the inertia force action increases; the total force increases first then decreases while the fill ratio is small and reach its peak at $\lambda=1.3$, the dominant role of inertial force is becoming more and more obvious, the total force is increasing after the fill ratio $\lambda \geq 1.3$, but no more than the maximum sloshing force at fill ratio of $\lambda=1$.

\subsection{The effect of lateral acceleration of fluid sloshing}

The lateral force of the tank car is affected not only by the fill ratio, but also by the lateral acceleration. This section selects the fill ratio $\lambda=1$, the $E I$ Centro ground motion as the 
motivation, calculating the inertial force, sloshing force, and total force. The lateral acceleration excitation is shown in Figure 3.

Figure 3 Lateral acceleration

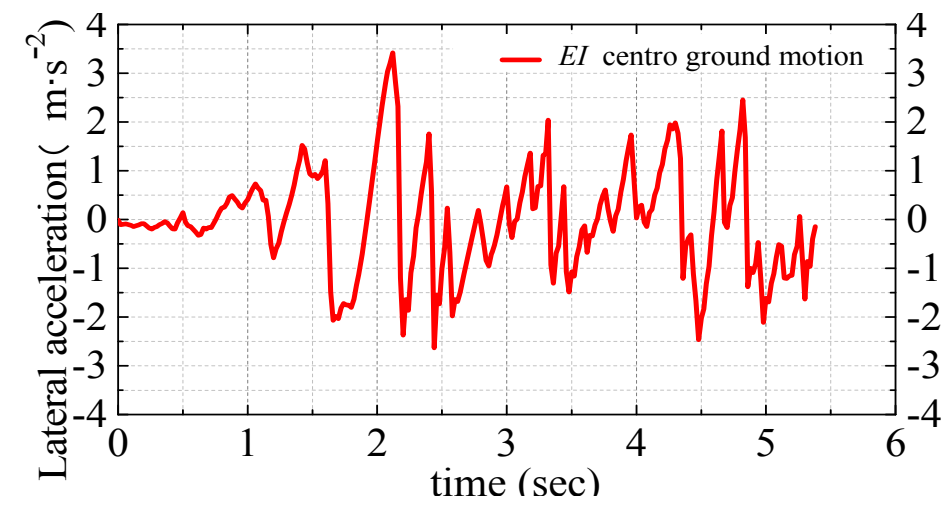

The inertial force, sloshing force, and total force are obtained with a truncation size $N=4$, the three forces are shown in Figures 4(a), 4(b) and 4(c).

Figure 4 Force diagram. (a) Inertial force (b) Sloshing force (c) Total force

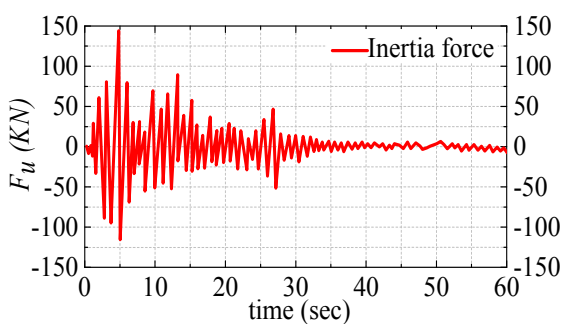

(a)

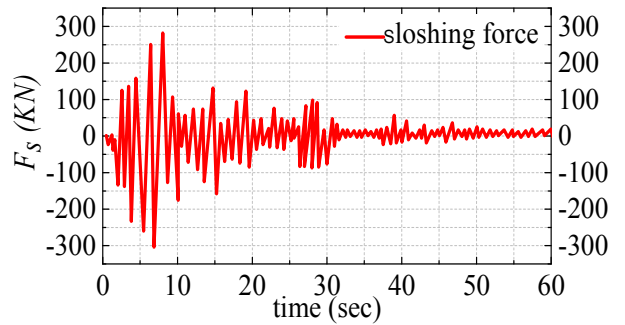

(b)

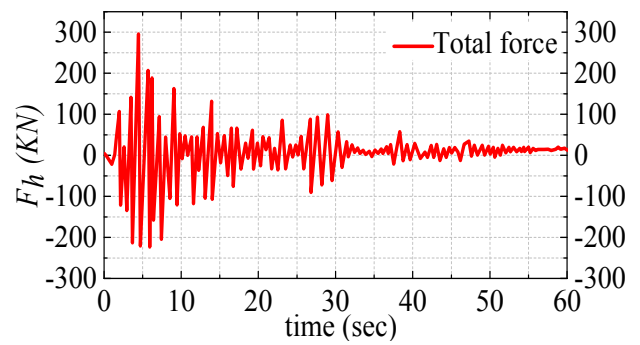

(c)

Figure 4(c) shows the maximum total force is $289 \mathrm{kN}$ at $4.4 \mathrm{~s}$, the sloshing force counteracts the inertia force and this is due to the fact that the dominant ground motion excitation frequencies are significantly larger than the dominant sloshing frequency (Papaspyrou et al., 2004). As the frequency of inertial force and sloshing force are 
different, the total force is not the algebraic sum of two values. Because liquid is not an ideal fluid, the kinetic energy of liquid dissipates as damping effect, and the inertial force and sloshing force approach to zero in the end, when the liquid stop shaking.

\section{The effect of liquid sloshing on vehicle motion}

Analyses of coupled fluid-vehicle systems in the presence of transient fluid slosh is known to be most challenging due not only to high computational demands of the CFD methods, but also to elaborate data transfer and coordinates transformations between the CFD and vehicle models. In a recent study, Shangguan et al. (2016) proposed a methodology for analysis of coupled fluid-vehicle system using the widely involved cosimulation of the TruckSim software. The methodology involved co-simulation of the TruckSim vehicle with a quasi-static fluid sloshing model, and provided an efficient tool to study effects of quasi-static slosh and the tank geometry on the vehicle roll stability. The transient fluid slosh effect thus could not be incorporated in the proposed method, while it was limited only to clean-bore tanks. In this study, the proposed fluid slosh model is coupled with the TruckSim vehicle model to study the effect of the transient fluid slosh on directional performance of the partly-filled tank vehicle. A co-simulation scheme is designed to evaluate time-varying slosh force and moment in Matlab/Simulink using the sloshing model, which is subsequently input to the TruckSim software to define considering equivalent rigid representation of the liquid load including the mass centre height of the cargo. Some important parameters of the vehicle are summarised in Table 1 and Figure 5 illustrates the co-simulation scheme, where slosh force and moment obtained from the fluid slosh model are considered as external inputs to the TruckSim model at each integration fluid slosh model for the subsequent time step. The lateral acceleration is obtained from the TruckSim, where the slosh force and moment are applied.

Table 1 Vehicle parameters

\begin{tabular}{lc}
\hline Tractor total mass & $8448 \mathrm{~kg}$ \\
Trailer total mass (empty tank) & $7997 \mathrm{~kg}$ \\
Maximum cargo mass (full tank) & $27120 \mathrm{~kg}$ \\
Centre of gravity height of the tractor sprung mass & $1.02 \mathrm{~m}$ \\
Centre of gravity height of the trailer sprung mass & $1.1 \mathrm{~m}$ \\
Tire track width of the tractor front axle & $2.03 \mathrm{~m}$ \\
Tire track width of the trailer front axle & $1.863 \mathrm{~m}$ \\
Tire track width of the trailer axle & $1.815 \mathrm{~m}$ \\
Tank centre height from the ground & $2.15 \mathrm{~m}$ \\
Tank length & $12 \mathrm{~m}$ \\
Tank radius & $1.5 \mathrm{~m}$
\end{tabular}

The response behaviour of five-axle vehicle with fluid $(\lambda=1)$ and same mass of solid are evaluated in step steering wheel input at constant speed of $40 \mathrm{~km} / \mathrm{h}$, the steering wheel input is shown in Figure 6. 
Figure 5 Coupled fluid-vehicle simulation model

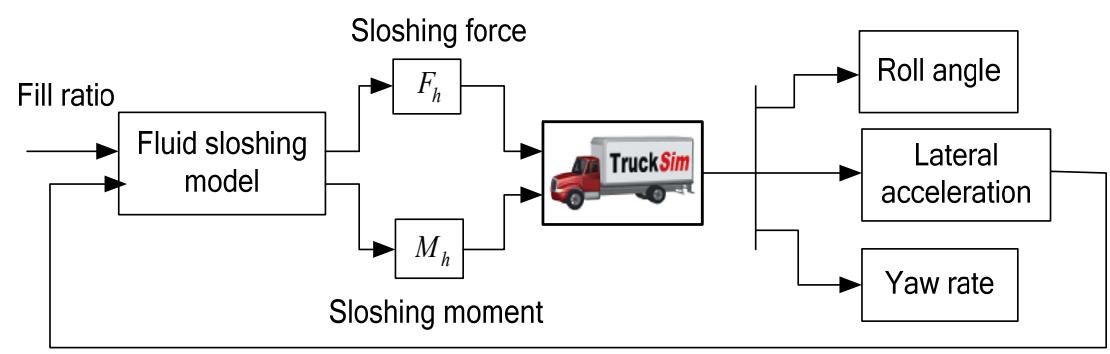

Figure 6 Steering angle

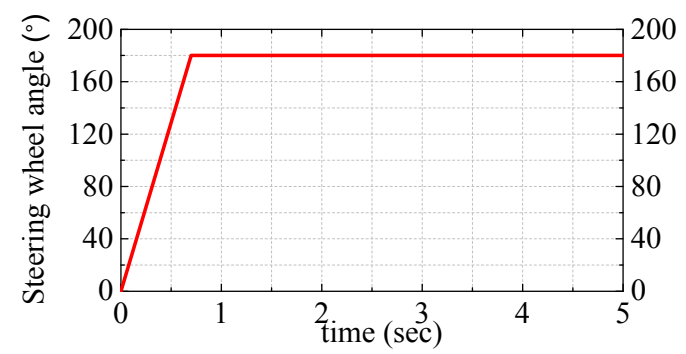

Two simulation tests of liquid and solid are carried out, the simulation results are shown in Figure 7.

Figure 7 Simulation curves of vehicle motion. (a) Lateral acceleration (b) Yaw rate (c) Roll angle

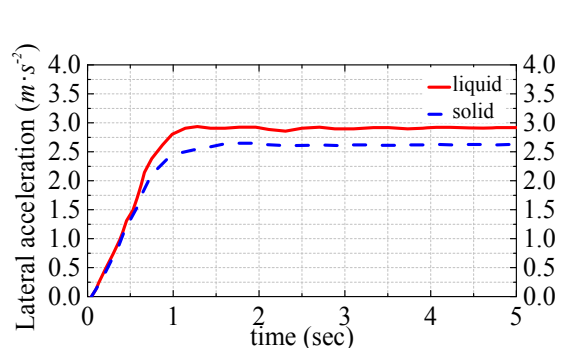

(a)

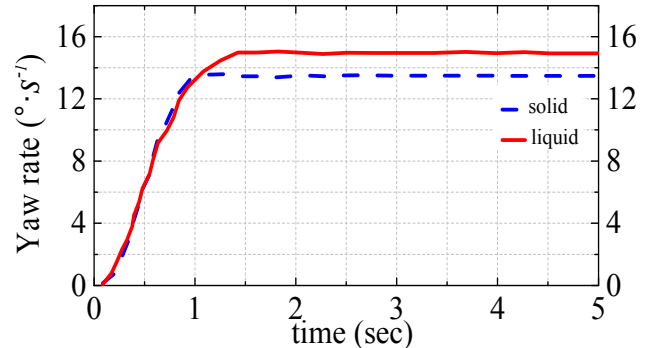

(b)

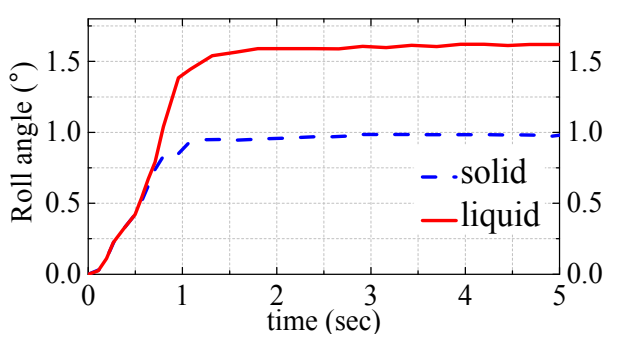

(c) 
The most significant rollover dynamic responses of the vehicle include yaw rate, yaw angle, roll angle, and lateral acceleration. As can be seen from Figure 7, the influence of liquid slosh on roll angle and yaw rate is obvious. This is because as the liquid moves, the fluid centre of gravity moves sideways, at the same time, the relative motion of tank generates dynamic pressure to increase the side inclination angle, and the roll angle and yaw rate increase, which are much bigger than the value of solid. The worse roll stability is because of fluid slosh and its interaction with vehicle dynamic, the stability of tank truck is obviously reduced.

\section{Kinetic model and simulation model of liquid tank semi-trailer}

\subsection{Kinetic model}

On horizontal road, disregarding the pitch and vertical motion, the character variation of tyres due to load change, when the wheel angle is small, the tyre lateral force can be represented by a linear tyre model. The structure of semi-trailer is shown in Figure 8.

Figure 8 Yaw and roll motion of tractor semi-trailer

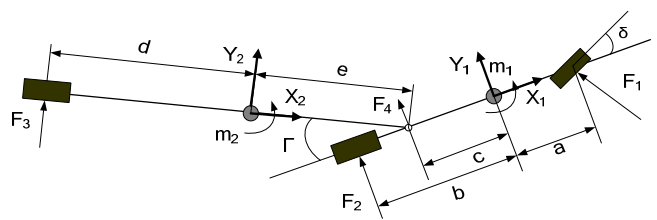

(a)

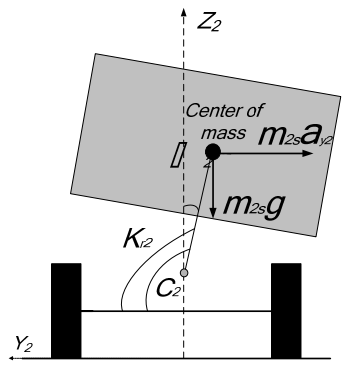

(b)

Kinematical equations of tractor

$$
\begin{aligned}
& m_{1} u_{1}\left(\dot{\beta}_{1}+\dot{\varphi}_{1}\right)-m_{1 s} h_{1} \ddot{\phi}_{1}=F_{1}+F_{2}+F_{4} \\
& I_{1 z z} \ddot{\psi}_{1}-I_{1 x z} \ddot{\phi}_{1}=F_{1} a-F_{2} b-F_{4} c \\
&\left.\quad I_{1 x x}+m_{1 s} h_{1}^{2}\right] \ddot{\phi}_{1}-I_{1 x z} \ddot{\psi}_{1}=m_{1 s} g h_{1} \phi_{1} \\
&+m_{1 s} h_{1}\left[u_{1}\left(\dot{\beta}_{1}+\dot{\psi}_{1}\right)-h_{1} \ddot{\phi}_{1}\right] \\
& \quad-k_{r 1} \phi_{1}-c_{1} \dot{\phi}_{1}+k_{12}\left(\phi_{2}-\phi_{1}\right)-F_{4} h_{1 c}
\end{aligned}
$$


Kinematical equations of semi-trailer

$$
\begin{aligned}
m_{2} u_{2}\left(\dot{\beta}_{2}+\dot{\psi}_{2}\right)-m_{2 s} h_{2} \ddot{\phi}_{2} & =F_{3}-F_{4} \cos \Gamma \\
I_{2 z z} \ddot{\psi_{2}}-I_{2 x z} \ddot{\phi_{2}}=-F_{3} d-F_{4} e \cos \Gamma & \\
{\left[I_{2 x x}+m_{2 s} h_{2}^{2}\right] \ddot{\phi_{2}}-I_{2 x z} \ddot{\psi_{2}}=} & m_{2 s} g h_{2} \phi_{2} \\
& +m_{2 s} h_{2}\left[u_{2}\left(\dot{\beta}_{2}+\dot{\psi}_{2}\right)-h_{2} \ddot{\phi}_{2}\right] \\
& -k_{r 2} \phi_{2}-c_{2} \dot{\phi}_{2}-k_{12}\left(\phi_{2}-\phi_{1}\right)+F_{4} h_{2 c}
\end{aligned}
$$

The kinematic constrain equation between the tractor and semi-trailer is obtained from equation (20).

$$
\dot{\beta}_{1}-\dot{\beta}_{2}-\frac{h_{1 c}}{u_{1}} \ddot{\phi}_{1}+\frac{h_{2 c}}{u_{2}} \ddot{\phi}_{2}-\frac{c}{u_{1}} \ddot{\psi_{1}}-\frac{e}{u_{2}} \ddot{\psi_{2}}+\dot{\psi}_{1}-\dot{\psi}_{2}=0
$$

\subsection{State space model}

The ideal sideslip angle and yaw rate of tractor and semi-trailer are defined as the state variables, front wheel angle is input, the state equation of degree of freedom (3-DOF) vehicle model handing characteristic in the process of turning can be expressed as:

$$
\left[\begin{array}{cccc}
\dot{\beta_{1 d}} & \dot{\varphi_{1 d}} & \dot{\beta_{2 d}} & \dot{\varphi_{2 d}}
\end{array}\right]^{T}=A\left[\begin{array}{llll}
\beta_{1 d} & \varphi_{1 d} & \beta_{2 d} & \varphi_{2 d}
\end{array}\right]^{T}+B \delta
$$

Because of the external interference, the actual sideslip angle and yaw rate deviate from the ideal sideslip angle and yaw rate. The adjustment of the semi-trailer motion can be achieved by imposing the additional transverse moment on tractor and trailer, the adjusted equation can be expressed as:

$$
\left[\begin{array}{llll}
\dot{\beta}_{1} & \dot{\varphi}_{1} & \dot{\beta}_{2} & \dot{\varphi}_{2}
\end{array}\right]^{T}=A\left[\begin{array}{llll}
\beta_{1} & \varphi_{1} & \beta_{2} & \varphi_{2}
\end{array}\right]^{T}+B \delta+B_{0} \Delta M
$$

Assume $x=\left(\begin{array}{llll}\Delta \beta_{1} & \Delta \varphi_{1} & \Delta \beta_{2} & \Delta \varphi 2\end{array}\right)^{T}, u=\Delta M$, the equation (22) minus equation (21) can be expressed as:

$$
\dot{x}=A x+B_{0} u
$$

Equation (23) reflects the dynamic relation between the pendulum moment and the state deviation. Linear Quadratic state Regulator (LQR) aim at linear time-invariant system, introducing the optimal control performance index $J, J$ is given by:

$$
J=\frac{1}{2} \int_{0}^{\infty}\left[x^{T}(t) Q x(t)+u^{T}(t) R u(t)\right] d t
$$


The control quantity $u(t)$ exists and unique, while index $J$ is mininmum. Namely

$$
u(t)=-R^{-1} B^{T} P x(t)
$$

Among $P$ can be got by solving the algebraic Riccati equation

$$
P A+A^{T} P-P B R^{-1} B^{T} P+Q=0
$$

Assume reaction coefficient $K=R^{-1} B^{T} P$,

$$
\Delta M=u(t)=-K x(t)
$$

The optimal yaw moment of semi-trailer is

$$
M_{T}=\Delta M \times \frac{J_{2}}{J_{1}}
$$

where $\beta_{1 d}, \phi_{1 d}, \beta_{2 d}, \phi_{2 d}, \beta_{1}, \phi_{1}, \beta_{2}, \phi_{2}$ are the ideal and reality side slip angle, yaw rate of tractor, semi-trailer, $\delta$ is the front wheel angle of tractor, $A, B$ are system matrix and input matrix, $u$ is yaw moment matrix, $Q$ is the weighted matrix of state variables, $R$ is the weighted matrix of input variables, $B_{0}=\left(\begin{array}{llll}0 & 1 / J_{1} & 0 & 0\end{array}\right)^{T}, J_{1}, J_{2}$ are the moment which rotation about centre of mass tractor and semi-trailer.

\section{The stability control of liquid tank semi-trailer}

In the extreme condition, the instability of tractor semi-trailer will lead to rollover accident. Aim at the liquid slosh reduce the stability of semi-trailer, the 3-DOF vehicle model is the reference model, the optimal yaw moment was calculated by optimal control theory, utilising the scheme of differential braking to assign the yaw moment, simulation in fishhook. The structure of the proposed active braking control scheme consists of two levels: the upper-level and the lower-level control. In the upper-level control, the desired yaw response for tractor and semi-trailer is obtained on the basis of the 3-DOF single track linear vehicle model, presented in the previous section. The corrective yaw moments required to track the reference model are computed using a $L Q R$ controller within the upper-level control. In the lower-level control, the brake torque distribution scheme is subsequently designed to produce the required yaw moment determined from the upper-level control. The control strategy diagram is shown in Figure 9.

Contrastive analysis with control and without control, the stability of semi-trailer in dangerous fill ratio and extreme condition. The lateral acceleration, roll angle, yaw rate, vertical load of the fifth axis of tractor are shown in Figures10(a), 10(b), 10(c) and $10(d)$. 
Figure 9 Schematic diagram of the optimal control system structure

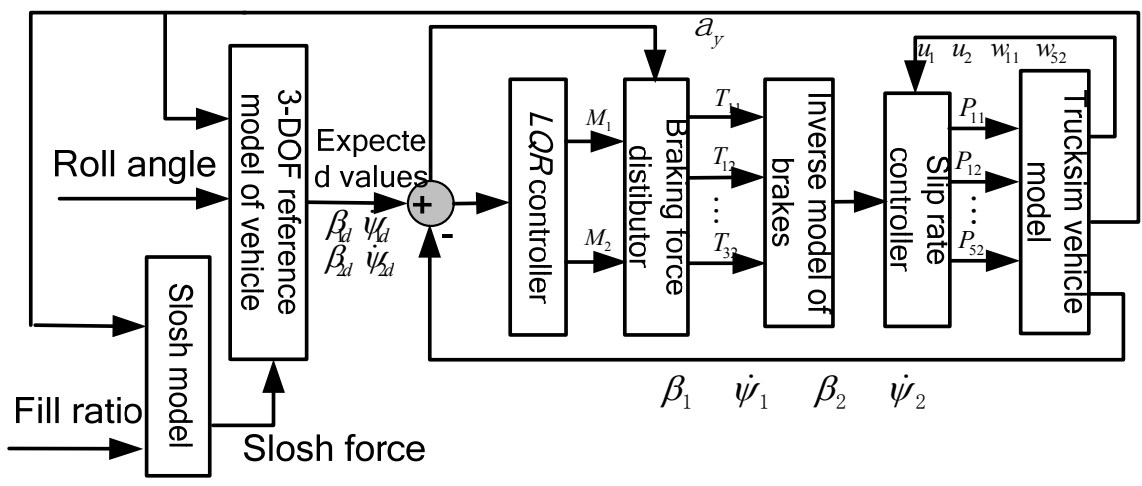

Figure 10 Operating parameters. (a) Lateral acceleration of tractor (b) Roll angle of tractor (c) Yaw rate of tractor (d) Vertical load of fifth axis of tractor

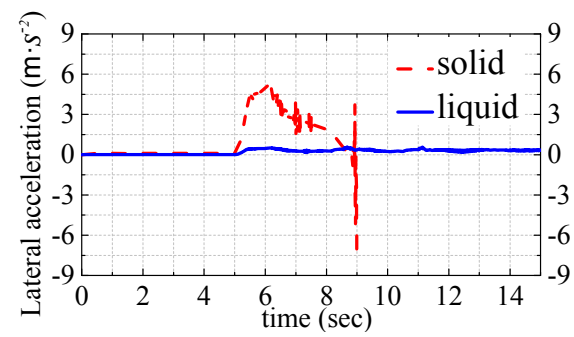

(a)

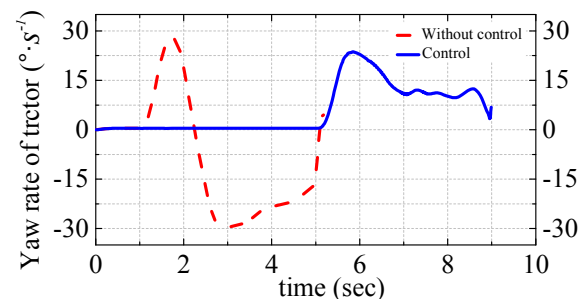

(c)

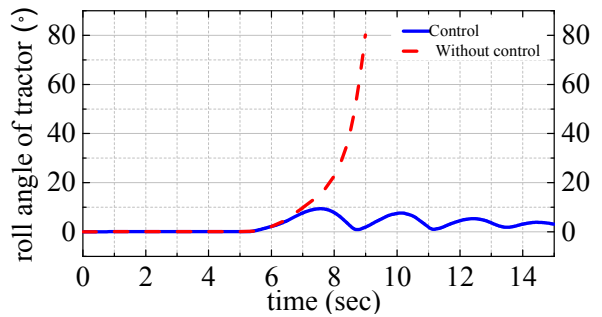

(b)

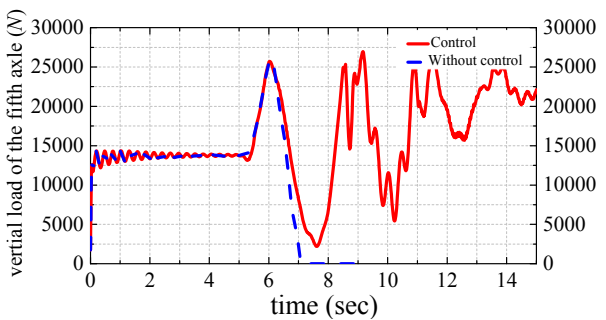

(d)

As can be seen from Figure 10, the yaw rate has wide range of fluctuations without control, the lateral acceleration caused by transverse motion, the lateral acceleration reach $0.75 \mathrm{~g}$ in $9 \mathrm{~s}$, exceed the lateral threshold value $0.6 \mathrm{~g}$ and it causes rollover, roll angle is $90^{\circ}$, the fifth axis tire goes off the ground, vertical load force is zero, it indicates that vehicle has gone rollover, however the vehicle with control do well, the lateral acceleration is controlled in $0.6 \mathrm{~g}$, roll angle is in $10^{\circ}$, the yaw rate is well controlled, the vertical load moves up and down in gravity, but it is stabilised in the end. 


\section{Conclusions}

In this work, the fluid slosh character is analysed with velocity potential, the effects of fluid slosh on liquid tractor semi-trailer is obtained are the coupled fluid-vehicle simulation model, the simulation results show that fluid sloshing reduces the stability of the vehicle. An active braking control strategy is proposed and analysed for prevention of tractor semi-trailer. A two-level control structure was synthesised, where the upper-level $L Q R$ controller is established from the required corrective yaw moment for the tractor semi-trailer, while the lower-level controller comprised a brake torque distribution scheme to decide which wheel(s) of tractor and or semi-trailer required braking to generate the required corrective yaw moments. The active braking control strategy is implemented on a TruckSim vehicle model to control the wheel braking torque of tractor and semi-trailer. From the simulation results, it is observed that the vehicle without control may roll over, while the vehicle with control shows good performance. The proposed controller could thus prevent the vehicle rollover under extreme conditions.

\section{Acknowledgements}

This research work was supported by the major science and technology public relations project in Jilin province about research on key technology and application of chassis performance improvement of micro car.

\section{References}

Ashtiani, I.H. et al. (2014) Effects of liquid cargo on lateral stability of B-Train combination, SAE Paper 2014-01-2319.

Azad, N.L. et al. (2009) 'A survey of stability enhancement strategies for articulated steer vehicles', International of Journal of Heavy Vehicle System, Vol. 16, No. 1, pp.26-48.

Bin, L. et al. (2015) Jackknifing Prevention of Tractor-Semitrailer Combination Using Active Braking Control, SAE Paper 2015-01-2746.

Cheli, F. et al. (2013) 'Simulation of sloshing in tank trucks', International of Journal of Heavy Vehicle System, Vol. 20, No. 1, pp.1-18.

Chen, C. and Tomizuka, M. (2000) 'lateral control of commercial heavy vehicle', International Journal of Vehicle Mechanics and Mobility, Vol. 33, No. 6, pp.391-420.

Goodarzi, A. et al. (2009) Integrated Yaw and Roll Moments Control for Articulated Vehicle, SAE Technical Paper 2009-01-2874.

Kang, X., Rakheja, S. and Stiharu, I. (2000) Directional Dynamic of a Partly-Filled Tank Vehicle Under Braking and Steering, SAE Technical Paper 2000-01-3477.

Oreh, S.H.T. et al. (2013) 'Directional control of articulated heavy vehicle', SAE International Journal, Vol. 6, No. 1, pp.143-149.

Oreh, S.H.T. et al. (2014) 'A sliding mode controller for directional control of articulated heavy vehicles', Journal of Automobile Engineering, Vol. 228, No. 3, pp.245-262.

Papaspyrou, S. et al. (2004) 'Response of half-full horizontal cylinders under transverse excitation', Journal of Fluids and Structures, Vol. 19, No. 4, pp.985-1003.

Salem, M.I. et al. (2009) 'Lateral sloshing in partially filled elliptical tanker trucks using a trammel pendulum', International of Journal of Heavy Vehicle, Vol. 16, No. 1, pp.207-224. 
Shangguan, W.B. et al. (2016) 'Simulation of a partially filled elliptical tanker trucks using a trammel pendulum', International of Journal of Heavy Vehicle System, Vol. 23, No. 1, pp.264-282.

Song, J. et al. (2007) 'Nonlinear observer and robust controller design for enhancement of vehicle lateral stability', Journal of Mechanical Science and Technology, Vol.21, No. 1, pp.98-105.

Yan, G. et al. (2009) 'Straight-line braking dynamic analysis of a partly filled baffled and unbaffled tank truck', Proceedings of the Institution of Mechanical Engineers, Vol. 223, No. 3, pp.11-26.

Zhao, W. et al. (2017) 'Roll response of an LNG carrier considering the liquid cargo flow', Ocean Engineering, Vol. 129, No. 2, pp.83-91. 